\title{
Preliminary study: myocardial T1 relaxation time in patients with ischemic findings and normal findings on coronary angiography
}

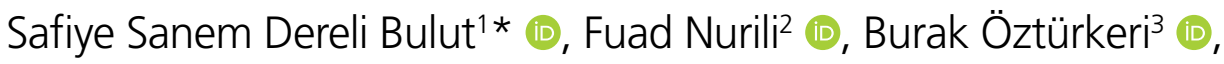

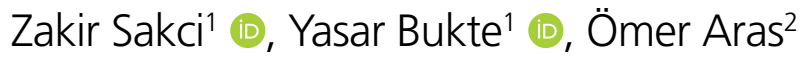

\begin{abstract}
SUMMARY
OBJECTIVE: The aim of this study is to evaluate the myocardium structure in patients with chest pain who were determined to have moderate and/or high risk for cardiac ischemic heart disease (IHD) but who had normal findings on conventional coronary angiography by using native cardiac magnetic resonance imaging (CMRI) T1 mapping and comparing with healthy volunteers.

METHODS: A total of 50 patients and 30 healthy volunteers who underwent CMRI were included in our prospective study. Patients whose clinical findings were compatible with stable angina pectoris, with moderate and/or high risk for IHD, but whose conventional coronary angiography was normal, were our patient group. Native T1 values were measured for 17 myocardial segments (segmented based on American Heart Association recommendations) by two radiologists independently. The data obtained were statistically compared with the sample $t$-test.

RESULTS: Myocardial native T1 values were found to be significantly prolonged in the patient group compared with the control group $(p<0.05)$. Inter-observer reliability for native T1 value measurements of groups was high for both patient and control groups $(\alpha=0.92$ for the patient group and 0.96 for the control group).

CONCLUSION: Findings suggestive of ischemia were detected by T1 mapping in the myocardium of our patients. For this reason, it is recommended that this patient group should be included in early diagnosis and close follow-up assessments for IHD.

KEYWORDS: Ischemic heart disease. Magnetic resonance imaging. T1 mapping.
\end{abstract}

\section{INTRODUCTION}

Chest pain is an important indicator of underlying ischemic heart disease (IHD). Differential diagnoses include other diseases of the mediastinum and/or thorax, as well as the upper abdomen $^{1,2}$. After clinical risk assessment, patients presenting with chest pain who are determined to be at moderate to high risk for IHD are advised to undergo functional tests [e.g., exercise electrocardiography (ECG), effort echocardiography (ECHO), single-photon emission computed tomography (SPECT), and scintigraphy) followed by coronary angiography ${ }^{1,2}$.

The presence of either ischemia or infarction on a stress imaging study is consistent with the diagnosis of IHD in a patient

\footnotetext{
'Health Sciences University, Istanbul Umraniye Training and Research Hospital, Department of Radiology - Istanbul, Turkey. ${ }^{2}$ Memorial Sloan Kettering Cancer Center, Department of Radiology - New York, USA.

${ }^{3}$ Health Sciences University, Istanbul Umraniye Training and Research Hospital, Department of Cardiology - Istanbul, Turkey.

*Corresponding author: ssanembulut@gmail.com

Conflicts of interest: the authors declare there is no conflicts of interest. Funding: none.

Received on December 21, 2020. Accepted on December 28, 2020.
} 
with angina symptoms. This is a clinical syndrome characterized by discomfort in the chest, jaw, shoulder, back, or arms, which is typically aggravated by exertion or emotional stress and relieved promptly with rest or by taking nitroglycerin ${ }^{1,2}$.

The American College of Cardiology (ACC) and the American Heart Association (AHA) devised a new score to estimate the 10-year risk of developing a first atherosclerotic cardiovascular disease (ASCVD) event, which was defined as non-fatal myocardial infarction (MI), coronary heart disease (CHD) death, or fatal or non-fatal stroke, in individuals who were initially free from $\mathrm{ASCVD}^{3}$.

Angina usually occurs in patients with CHD but also it can occur in individuals with valvular disease, hypertrophic cardiomyopathy, and uncontrolled hypertension ${ }^{3,4}$. Infrequently, patients with normal coronary arteries may experience angina related to coronary spasm or endothelial dysfunction ${ }^{4}$.

A subset of patients will have no findings of coronary artery pathology on conventional coronary angiography despite having $\mathrm{MI}^{5}$.

Coronary heart disease (CHD) also includes a microvascular component. This process includes impaired endothelial function, inflammation, neovascularization, apoptosis, and the hypercoagulable state ${ }^{4,5}$.

Cardiac microvascular dysfunction (CMD) is the impaired ability of microcirculation of the heart to adapt blood flow to meet oxygen demand. Microvascular disease should be suspected when chest pain persists after physical effort and shows slow or no response to short-acting nitrates ${ }^{4,5}$.

The increasing number of both basic research and clinical studies, particularly those that develop novel imaging techniques, are promising for detection of early microvascular functional changes and early changes in the myocardium ${ }^{5}$.

Over the past decades, new magnetic resonance imaging (MRI) sequences have been developed, which allow pixel-wise longitudinal relaxation time (T1) mapping of the myocardium. Native T1 mapping is emerging as an important tool for characterizing myocardial tissue with higher accuracy than nonspecific functional parameters ${ }^{6,7}$.

Cardiovascular magnetic resonance research in animals and humans has shown that native T1 values (i.e., pre-contrast T1) are prolonged in pathologies, such as edema, infarction, amyloid infiltration, and fibrosis, and shortened in the presence of fat and iron accumulation ${ }^{7-10}$. The native $\mathrm{T} 1$ signal from the left ventricular myocardium has shown a particular promise for non-invasively differentiating diseased from normal regions ${ }^{11,12}$.

In acute MI, T1 increases are more pronounced in myocardium that will become infarcted than in myocardium which will be salvaged by reperfusion. Following reperfusion, T1 increases further in infarcted tissue, but remains unchanged in salvaged myocardium ${ }^{12}$.

In this study, we aimed to evaluate the myocardium structure by applying the Saturation Method Using Adaptive Recovery Times for Cardiac T1 Mapping (SMART Map) technique (1.5 T, Optima MR450w, GE Healthcare, Waukesha, WI, USA) in patients who underwent cardiac assessment and cardiac function tests (i.e., effort test and cardiac scintigraphy) and were determined to have moderate to high risk for IHD who had normal findings on conventional coronary angiography. We compared our findings on these patients with that of healthy controls.

\section{METHODS}

This prospective study was approved by the ethical committee at our hospital. The verbal and written informed consent was obtained from all participants.

\section{Patient population}

Between December 2016 and March 2019, we included a total of 50 patients ( 27 female and 23 male patients) with chest pain who had moderate and/or high risk for IHD but who had normal conventional coronary angiography findings.

\section{Patient evaluation before magnetic resonance scan}

Risk assessment of ASCVD was made according to the guidelines 5 .

Trained staff measured height, weight, and supine blood pressure using a standard mercury sphygmomanometer and serum total cholesterol ratio (TCR). The serum TCR was calculated by dividing the total cholesterol number by high-density lipoprotein (HDL) number from a non-fasting blood sample. This ratio was between 4 and 1 . Higher ratios mean a higher risk for heart disease.

Participants completed a questionnaire about their demographic characteristics, smoking history (i.e., never, former, or current smoking), medical diagnoses, and treatments (including hypertension and diabetes).

Typical angina pectoris criteria were as follows: substernal chest pain or a feeling of discomfort, increased pain with activity or emotional stress, and reduced pain with rest or nitroglycerin.

Those patients with typical angina complaints were included in this study.

Patients with evidence of acute coronary artery disease (CAD) detected in the laboratory analysis (e.g., troponin elevation) and with pathology and/or disease (e.g., diabetes mellitus, valve diseases, hypertension, cardiomyopathies, amyloidosis, systemic diseases, acute-chronic MI, myocarditis, etc.) 
that could cause changes in the myocardial native $\mathrm{T} 1$ time were excluded. In addition, patients with a contraindication to MRI (e.g., pacemaker, stent in coronary arteries, pregnancy, and claustrophobia) or who had intravenous (IV) stents and similar equipment that could affect image quality were excluded.

\section{Evaluation of patients' resting electrocardiograms}

Resting ECGs were classified as showing major, minor, or no abnormalities.

Minor ECG abnormalities for our patient group are as follows: first- and second-degree atrioventricular block, borderline prolonged ventricular excitation, frequent atrial or ventricular premature beats, and fascicular blocks.

Major ECG abnormalities for our patient group are as follows: atrial fibrillation or atrial flutter, high-degree atrioventricular dissociation, left bundle-branch block, right bundle-branch block, indeterminate conduction delay, Q-wave MI, and isolated ischemic abnormalities.

The 12-lead ECG recordings were obtained in all cases. Stress ECGs were evaluated as non-ischemic, ischemic, and non-diagnostic. Horizontal or down sloping depression of $\geq 1 \mathrm{~mm}$ in patients without ST depression at rest and 1-mm additional collapse in patients with ST depression at rest were considered ischemia. If additional collapse was $<1 \mathrm{~mm}$ in patients with ST depression at rest, it was considered non-diagnostic.

\section{Evaluation of patients' echocardiography}

After clinical and laboratory evaluation, echocardiography was performed on patients for ventricular functions. Patients with impaired cardiac wall movements and with left ventricular ejection fraction (EF) below 50\% were excluded from this study.

\section{Coronary angiography}

Coronary angiography was planned for artery evaluation in patients with ischemia findings after stress tests. Normal coronary angiography was considered as smooth vessel lumen wall and absence of obstructive lesions.

Patients with normal coronary angiography findings and abnormal stress test findings were included in this study.

The mean time between coronary angiography and cardiac magnetic resonance imaging (CMRI) was 1 week.

\section{Control group}

In our control group, there were 30 healthy volunteers ( 18 female and 12 male patients) without any cardiac or systemic disease.

Echocardiography and ECGs were normal in our control group.
No conventional coronary angiography was performed in the patients in our control group.

\section{Cardiac magnetic resonance imaging}

All patients and control volunteers underwent MRI in the supine position on a 1.5-T MR scanner (Optima MR450w, GE Healthcare) with a 32-channel cardiac coil.

\section{Image protocol of cardiac magnetic resonance examination}

The routine CMRI sequences of our institution were as follows: trans-axial $(8-10 \mathrm{~mm})$ set of T2-weighted fast spin echo images through the chest. Steady-state free precession (SSFP) cine imaging with short-axis plane from the mitral valve plane through the apex of the heart (with slice thickness of 6-8 $\mathrm{mm}$, 2-4 mm inter-slice gaps to equal $10 \mathrm{~mm}$, and temporal resolution of $\leq 45 \mathrm{~ms}$ between phases) and SSFP cine imaging with long-axis plane in vertical and horizontal long-axis planes were obtained through the apex and center of the mitral and tricuspid valves (i.e., a set of four more rotational long-axis views were obtained).

After routine CMRI, all patients and control volunteers underwent pre-contrast short-axis SMART ${ }_{1}$ Map imaging of the left ventricle for this study.

The SMART 1 Map imaging involved the acquisition of three slices (i.e., basal, mid-ventricular, and apical of heart) at five saturation delay times over a scan time of nine heartbeats (i.e., requiring 1, 1, 1, 2, and 4 heartbeats, respectively). Short saturation recovery times ( $T S s<R R$ interval) were acquired within a single heartbeat and were automatically distributed between TS $\mathrm{min}$ and trigger delay. Longer TSs (free relaxation up to $4 \times R R$ ) were performed across multiple heartbeats. An additional image corresponding to an infinite delay time $(\mathrm{TS}=\infty)$ was acquired without saturation. SMART Map imaging (repetition time/ echo time, TR/TE=2.8/1.2 ms; TS=100-4000 ms; flip angle, $\mathrm{FA}=45$; field-of-view, $\mathrm{FOV}=38 \times 30 \mathrm{~cm}$; slice thickness $/$ spacing $=8 / 5 \mathrm{~mm}$; bandwidth, $\mathrm{BW}=125 \mathrm{kHz}$; matrix $=160 \times 128$ ) was performed under breath hold.

Cardiac magnetic resonance (CMR) scan of patient group was performed by the IV contrast agent administration in $0.2 \mathrm{~mL} / \mathrm{kg}$ dose (gadoteric acid, $0.5 \mathrm{mmol} / \mathrm{mL}$, Guerbet ${ }^{\circledR}$ ) for myocardial delayed enhancement (MDE) imaging.

Cardiomyopathies, amyloidosis, systemic diseases, acutechronic MI, myocarditis, etc. could cause abnormal myocardial contrast enhancement in late phase in CMR. Therefore, patients with pathological myocardial contrast enhancement typical for these disorders in late phase MDE images were excluded from the study. 
There was no IV contrast agent administration in our control group.

\section{Image analysis and post processing}

The cardiac magnetic resonance (CMR) analysis was performed independently by two radiologists (with experience in CMR for 10 and 8 years, respectively) in two sessions.

Morphological and functional evaluation of the heart was performed in the first session. Cardiac function analysis was done automatically in workstation (CardiacVX, GE Healthcare).

All short-axis SMART Map images and 2D color maps were evaluated with computer-aided analysis packages for planimetry of endocardial and epicardial borders at end-diastole in the second session. Myocardial native T1 values were measured.

Short-axis SMART 1 Map images of the left ventricle were obtained at apical, mid-ventricular, and basal levels. Segmentation of the left ventricle was done as recommended by the AHA. Region of interest (ROI) placement was performed on native SMART $_{1}$ Map, which was automatically reconstructed and superimposed on the 17-Segment Model of the AHA's standard left ventricular anatomy and was performed by drawing the endocardial and epicardial contours of the left ventricular myocardium. Myocardial native T1 values for a total of 16 segments (excluding the apex) were measured after ensuring that the ROI was within the limits of myocardium (Figure 1a-c).
According to the measurement results, if prominent prolongation in native $\mathrm{T} 1$ value was detected (considered statistically outlier), they were excluded (Figures 1a and 1c).

In addition, both radiologists also evaluated T1 mapping image quality. Scoring of this category is as follows:

$1=$ unable to see;

$2=$ blurry but visualized;

$3=$ acceptable;

4 =good; and

$5=$ excellent.

\section{Statistical analysis}

The distribution of outcome categories was assessed using the Shapiro-Wilk test. The data were presented as average \pm standard deviation, based on the normality of the data. In the statistical analysis, outlier values were excluded so that they do not affect the average value of native $T 1$. The categorical variables were reported as counts and percentages. The paired sample $t$-test was performed to compare the differences between groups. The Cronbach's alpha value was used to assess inter-observer reliability for myocardial native $\mathrm{T} 1$ measurements. The statistically significant $p$-value was accepted as $<0.05$. All assessments were performed using the Statistical Package for the Social Sciences (SPSS) software (version 22.0; SPSS Inc., Chicago, IL, USA).
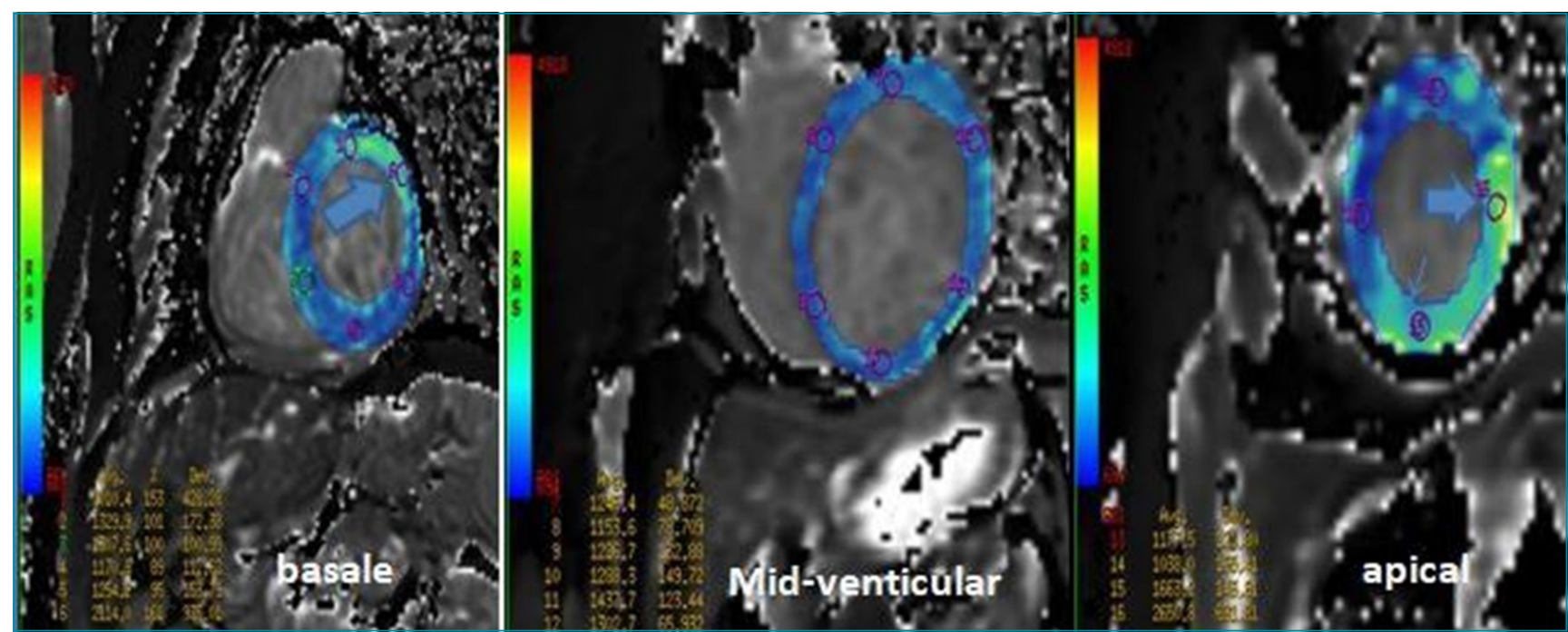

Figure 1. Images obtained from the study of a 46-year old male patient with chest pain and active smoker (i.e., average 1.5 packs/day) with a total cholesterol ratio value of 3 . The native T1 value measurements were made from short-axis sections obtained from (A) the basal, (B) midventricular, and (C) apical levels of the heart. Myocardium is shown in blue color after the endocontour (i.e., blue-colored thin arrow on the apical segment) and epicontour are drawn. The native T1 values corresponding to each segment according to the American Heart Association classification of automatic segmentation are measured for circular region of interest in the myocardium. The obtained values can be seen in the left lower corner of the pictures (i.e., average 1094.8 ms). Prominent prolongations in native T1 values (2914 and 2500 ms) were detected that were considered statistically outlier, and so they were excluded (blue-colored thick arrows). 


\section{RESULTS}

A total of 50 patients (27 female and 23 male patients) with ischemic findings but normal conventional coronary angiography and 30 healthy volunteers (18 female and 12 male patients) were included in this study.

The age range of the patient group was between 35 and 62 years (mean age $=45$ ). The heart rate measured during the CMR examination was between 60 and 84 heartbeats/minute (HB/min). The mean BMI was $27 \mathrm{~kg} / \mathrm{m}^{2}$.

The age range of the control group was between 30 and 55 years (mean age $=48$ ) without any cardiac or systemic disease. The heart rate measured during this study was between 75 and $82 \mathrm{HB} / \mathrm{min}$. The mean BMI was $24 \mathrm{~kg} / \mathrm{m}^{2}$.

Total cholesterol ratio assessment of our patient group was as follows: ratio $1=16$ patients, ratio $2=14$ patients, ratio $3=17$ patients, and ratio $4=3$ patients. Twenty-one patients in our group were active smokers (i.e., average 2 packs/day). After familial history evaluation, 15 patients revealed to have diabetes mellitus and 19 patients have hypertension in their relatives. No abnormality was present in 16 patients during ECG examination but 4 patients had minor changes. Those changes were frequent atrial or ventricular premature beats.

The mean EF values for both patient (left ventricle average EF $62 \%$ and right ventricle average EF 52\%) and control volunteer (left ventricle average EF 65\% and right ventricle average EF 54\%) groups were within normal limits.

Digital subtraction angiography (DSA) coronary angiography was normal in the patient group. There was no evidence of stenosis in the coronary arteries. There were smooth vessel lumen walls.

Heart muscle contractions were natural on functional evaluation, in both patients and volunteers. Cusp functions were normal and no evidence of insufficiency and/or stenosis was detected.

Figures 2 and 3 show the native T1 values in the patient group and control group as performed by the first and second radiologists, respectively.

Average native $\mathrm{T} 1$ values were significantly longer in the patient group than in the control group $(\mathrm{p}<0.05)$. This difference was less pronounced in segments $3,9,13$, and 14 (AHA) $(\mathrm{p}=0.04, \mathrm{p}=0.045, \mathrm{p}=0.042$, and $\mathrm{p}=0.038$, respectively) while other segments were more prominent $(\mathrm{p}<0.001)$.

When $\mathrm{T} 1$ mapping was evaluated in terms of image quality, both observers evaluated the image quality as good (score $=4$ ) with high compliance (alpha=0.87).

\section{DISCUSSION}

In this study, we aimed to evaluate the myocardial structure using the SMART ${ }_{1}$ Map technique in patients who underwent cardiologic assessment due to chest pain and who were found to have moderate to high risk for IHD in stress tests, but who had normal conventional coronary angiography. We compared our findings with that of healthy participants.

Coronary artery disease (CAD) also includes a microvascular component. CMD is the impaired ability of microcirculation of the heart to adapt blood flow to meet oxygen demand ${ }^{13}$.

Microvascular disease should be suspected when chest pain persists after physical effort and shows slow or no response to short-acting nitrates ${ }^{13}$. This was the case in our patient group.

Transthoracic Doppler echocardiography (TTDE) is an initial screening method to detect significant microcirculation impairment. It allows the measurement of coronary blood flow

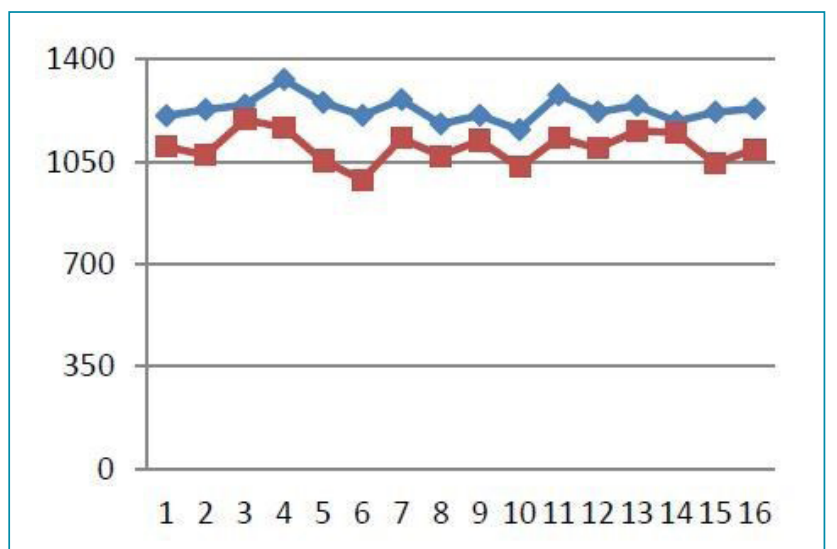

Figure 2 . The graph shows the native T1 values as measured by the first observer for the patient group (blue curve) and the control group (red curve). The native T1 values of the patient group were significantly longer $(p<0.001)$. The values along the $x$-axis show the native T1 signal values and the measured cardiac segments along the $y$-axis.

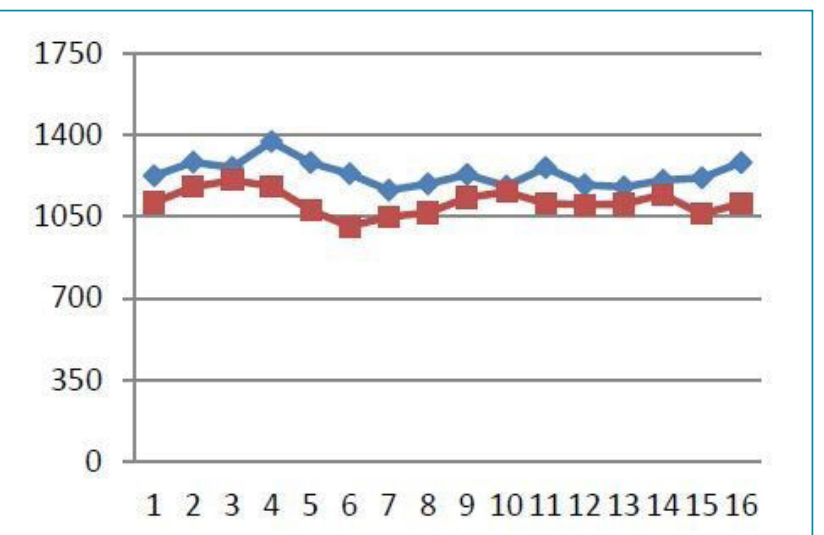

Figure 3. The graph shows the native T1 values as measured by the second observer for the patient group (blue-colored curve) and the control group (red-colored curve). The native T1 values in the patient group were significantly longer $(p<0.001)$. The values along the $x$-axis show the native T1 signal values and the measured cardiac segments along the $y$-axis. 
$(\mathrm{CBF})$ velocity in the left anterior descending artery. With this method, coronary microvascular dilatator function is calculated as the ratio of diastolic $\mathrm{CBF}$ velocity at peak vasodilatation to CBF velocity at rest ${ }^{13,14}$.

Mild coronary microvascular dysfunction may not be identified earlier by TTDE, and microvascular dysfunction can be assessed only in the left anterior descending artery perfusion area, because other coronary arteries are not well visualized ${ }^{14}$.

In addition, diastolic left ventricular dysfunction is a first sign of myocardial changes even in younger patients with normal systolic function detected by transthoracic echocardiography $(\mathrm{TTE})^{14}$. In our patients, we found that TTE showed early signs of diastolic dysfunction.

The endothelium is a central regulator of vascular homeostasis. This includes hormone transport and distribution, metabolic waste product disposal and regulation of regional blood flow by synthesis, and release of different mediators with opposing vascular properties ${ }^{13,14}$.

Metabolic abnormalities (i.e., hyperglycemia, hyperlipidemia, insulin resistance, and insulin deficiency) are lead to myocardial fibrosis and/or myocardial hypertrophy directly or indirectly. Several processes are responsible for these adverse changes including impaired calcium cycling, myocardial insulin resistance, increased lipid uptake, glucotoxicity, and activation of the renin-angiotensin-aldosterone system observed in mouse models of dilated cardiomyopathy ${ }^{13,14}$.

Increased activation of the renin-angiotensin-aldosterone pathway leads to the fibrosis formation. These processes lead to altered myocardial relaxation and manifest as diastolic dysfunction. Systolic dysfunction is a later manifestation, usually occurring after diastolic dysfunction develops ${ }^{14,15}$.

Neurogenic, hormonal, metabolic, myogenic, and flow (shear stress-induced) endothelial mechanisms control coronary vascular resistance at specific microvascular sites. Integration of multiple mechanisms could hypothetically form a system matching CBF to myocardial metabolic demands ${ }^{13-15}$.

A unique feature of CMR is its ability to characterize myocardium. Proton relaxation times, T1, T2, and T2*, are a reflection of the composition of individual tissues and change in the presence of disease ${ }^{16,17}$. Research into T1 mapping has largely been focused on the study of cardiomyopathies, but T1 mapping also shows huge potential in the study of IHD ${ }^{18}$.

Systemic disorders that affect the heart, such as sarcoidosis, systemic lupus erythematosus, and systemic sclerosis, are known to induce tissue edema at the acute stage and exhibit elevated T2 (and T1) values. Thus, T1 mapping may be a useful clinical tool for identifying cardiac involvement with implications for therapy and for assisting in determination of prognosis ${ }^{15-17}$.
Beyond focal lesions that are well depicted by late gadolinium enhancement sequences, T1 mapping has made it possible to discriminate diffuse myocardial alterations, previously not accessible by other non-invasive means. The strengths of T1 mapping are high reproducibility and immediate clinical applicability, even without the use of contrast media injection (i.e., native $\mathrm{T} 1)^{15,16}$.

T1 mapping is allowed for the non-invasive quantitative assessment of cardiac remodeling. Native T1 mapping has shown pathology with high accuracy in cases of IHD, acute MI, and myocardial edema, even demonstrating a higher sensitivity than T2-weighted short-tau inversion recovery (IR) imaging ${ }^{16,18}$.

Cardiac T1 mapping methods are performed either by IR or saturation recovery (SR) technique, followed by pre-pulse sequences applied on this basis, resulting in raw images generated by different degrees of recovery along the z-axis. Then, two-dimensional color T1 maps are created automatically ${ }^{19}$.

The most common T1 mapping strategy involves an IR pulse sequence, in which the application of a non-section selective $180^{\circ}$ pulse inverts the magnetization. As longitudinal magnetization recovers, at a time defined as the inversion time (TI), a section-selective $90^{\circ}$ excitation pulse is applied and rotates the magnetization into the transverse plane. This is repeated again and again, each time with the TI changed. This method, which is very sensitive to many patient-related factors (i.e., heart rate, obesity, and blood hematocrit value), especially the changes that may occur in R-R intervals, has been modified in various ways ${ }^{18-20}$.

The Modified Look-Locker Inversion Recovery (MOLLI) method, which was first developed as a Look-Locker technique, was developed with a single-shot balanced SSFP to reduce scan time and artifact sensitivity.

There are IR pulse sequences, such as shortened MOLLI (shMOLLI), which are developed for signal collection, differences in breathing techniques, and breathless patients. With these methods, the apparent $\mathrm{T} 1$ value of the myocardium can be calculated ${ }^{10-12}$.

However, in this study, we used the SMART Map technique, which uses the SR preparation instead of the IR technique, and is similar to the saturation recovery single shot acquisition technique. Dephasing the whole imaging volume leads to depletion of the entire magnetization, alleviating the need for any rest periods ${ }^{19,20}$. In this way, we could measure the true $T 1$ value of the myocardium by the SMART ${ }_{1}$ Map technique, regardless of the patient-related or device-related effects ${ }^{20}$. When we evaluated the studies performed in the literature, we found that there were not enough in vivo studies with the SMART Map technique for myocardium ${ }^{11-20}$. 
The normal natural myocardial $\mathrm{T} 1$ values measured at $1.5 \mathrm{~T}$ with MOLLI in the literature are between 900 and $1100 \mathrm{~ms}$, but as we have already mentioned, it depends on several technical and physiological parameters ${ }^{20}$. In this study, the mean native $\mathrm{T} 1$ value of myocardium was $1101 \pm 89$ according to the measurements in the control group and was in concordance with the literature.

We believed that the prolongation we have detected in the myocardial native T1 value with the SMART Map method in our group is caused by ischemic damage. This finding can be deemed reversible since no heart contraction dysfunction accompanies this segmental or diffuse myocardial native $\mathrm{T} 1$ prolongation in our patient group ${ }^{18-}$ ${ }^{20}$. We believed that the native $\mathrm{T} 1$ prolongation we have detected in our study is caused by transient myocardial edema and inflammation ${ }^{16,18}$.

\section{Limitations}

There are some limitations of this study. First, the fact that native $\mathrm{T} 1$ value difference between patient and control groups in segment 3, 9, 13, and 14 in accordance with AHA classification (i.e., basal inferoseptal, mid-inferoseptal, apical anterior, and apical septal, respectively) is thought to be related to secondary to the artifact caused by the actuality that these segments are much more mobile compared to the other parts of the heart (e.g., closer proximity to diaphragm). Perhaps to prevent this, operators must aim to minimize partial volume effects by optimal slice orientation relative to the tissue, which is preferably orthogonal to the imaging plane to minimize obliquity ${ }^{10,17,18}$.

Second, we did not make extracellular volume fraction measurements. The normal myocardium consists of two parts: the intracellular compartment (including myocytes, fibroblasts, and other blood cells such as red cells) and the extracellular compartment (dominant water associated with the extracellular matrix, but also including the intracapillary plasma volume). The native $\mathrm{T} 1$ value notifies us about both compartments. Extracellular volume fraction measurements may show changes in the extracellular compartment by allowing hematocrit correction, which may allow the origin of the pathology to be elucidated ${ }^{21}$.

In addition, as a preliminary study, the number of patients and healthy people in the control group was small, and more extensive work will be needed to verify our findings.

\section{CONCLUSIONS}

This study shows that in the patients with clinical findings as stable angina with abnormal stress test results, the prolonged myocardial native T1 value was observed even if conventional coronary angiography was normal. When evaluated together with clinical findings, this should be considered significant in terms of microvascular dysfunction and should be included in early diagnosis and follow-up assessments for IHD.

\section{ACKNOWLEDGMENTS}

We thank the radiologists Cengiz Han Cavli and Azim Celik (Regional Research Manager at GE Healthcare, Austria GmbH $\&$ Co OG) for their contributions.

\section{ETHICAL APPROVAL}

This article does not contain any studies with animals performed by any of the authors. This study had an ethical committee approval from the local institution (no. 15718). Informed consent was obtained from all individual participants included in this study.

\section{AUTHORS" CONTRIBUTIONS}

SSDB: Conceptualization, Data curation, Formal analysis, Writing - original draft. FN: Data curation, Formal analysis, Writing - original draft. BO: Data curation, Formal analysis. ZS: Data curation, Formal analysis. YB: Writing - review \& editing. OA: Writing - review \& editing.

\section{REFERENCES}

1. Ammann P, Marschall S, Kraus M, Schmid L, Angehrn W, Krapf $R$, et al. Characteristics and prognosis of myocardial infarction in patients with normal coronary arteries. Chest. 2000;117(2):333-8. https://doi.org/10.1378/chest.117.2.333

2. Florian A, Jurcut R, Ginghina C, Bogaert J. Cardiac magnetic resonance imaging in ischemic heart disease: a clinical review. J Med Life. 2011;4(4):330-45. PMID: 22514564
3. Wilson PWF, Polonsky TS, Miedema MD, Khera A, Kosinski AS, Kuvin JT. Systematic review for the 2018 AHAVACC/AACVPR/ AAPAABC/ACPM/ADAVAGS/APhA/ASPC/NLAPCNA guideline on the management of blood cholesterol: a report of the American College of Cardiology/American Heart Association Task Force on Clinical Practice Guidelines. J Am Coll Cardiol. 2019;73(24):321027. https://doi.org/10.1016/j.jacc.2018.11.004 
4. Kibel A, Selthofer-Relatic K, Drenjancevic I, Bacun T, Bosnjak I, Kibel $D$, et al. Coronary microvascular dysfunction in diabetes mellitus. J Int Med Res. 2017;45(6):1901-29. https://doi. org/10.1177/0300060516675504

5. Bekkers CAM, Yazdani SK, Virmani R, Waltenberger J. Microvascular obstruction underlying pathophysiology and clinical diagnosis. J Am Coll Cardiol. 2010;55(16):1649-60. https://doi.org/10.1016/j.jacc.2009.12.037

6. Radenkovic D, Weingärtner S, Ricketts L, Moon JC, Captur G. T1 mapping in cardiac MRI. Heart Fail Rev. 2017;22(4):415-30. https://doi.org/10.1007/s10741-017-9627-2

7. Reitera $G$, Reiterb $C$, Kräuter $C$, Fuchsjägerb $M$, Reiter U. Cardiac magnetic resonance T1 mapping. Part 1: aspects of acquisition and evaluation. Eur J Radiol. 2018;109:223-34. https://doi.org/10.1016/j.ejrad.2018.10.011

8. Messroghli DR, Walters K, Plein S, Sparrow P, Friedrich MG, Ridgway JP, et al. Myocardial T1 mapping: application to patients with acute and chronic myocardial infarction. Magn Reson Med. 2007;58(1):34-40. https://doi.org/10.1002/mrm.21272

9. Oh-lci D, Jeuthe S, Al-Wakeel N, Berger F, Kuehne T, Kozerke $S$, et al. T1 mapping in ischaemic heart disease. Eur Heart J Cardiovasc Imaging. 2014;15(6):597-602. https://doi. org/10.1093/ehjci/jeu024

10. Schelbert EB, Messroghli DR. State of the art: clinical applications of cardiac T1 mapping. Radiology 2016;278(3):658-76. https:// doi.org/10.1148/radiol.2016141802

11. Hwang SH, Choi BW. Advanced cardiac MR imaging for myocardial characterization and quantification : T1 mapping. Korean Circ J. 2013;43(1):1-6. https://doi.org/10.4070/ kcj.2013.43.1.1

12. van Assen $M$, van Dijk R, Kuijpers D, Vliegenthart R, Oudkerk $M$. T1 reactivity as an imaging biomarker in myocardial tissue characterization discriminating normal, ischemic and infarcted myocardium. Int J Cardiovasc Imaging. 2019;35(7):1319-25. https://doi.org/10.1007/s10554-019-01554-4

13. Komaru T, Kanatsuka H, Shirato K. Coronary microcirculation: physiology and pharmacology. Pharmacol Ther. 2000;86(3):21761. https://doi.org/10.1016/s0163-7258(00)00057-7
14. Perez-Terol I, Rios-Navarro C, Dios E, Morales JM, Gavara J, Perez-Sole N, et al. Magnetic resonance microscopy and correlative histopathology of the infarcted heart. Sci Rep. 2019;9(1):20017. https://doi.org/10.1038/s41598-019-56436-5

15. Iles L, Pfluger H, Phrommintikul A, Cherayath J, Aksit P, Gupta $S N$, et al. Evaluation of diffuse myocardial fibrosis in heart failure with cardiac magnetic resonance contrast-enhanced T1 mapping. J Am Coll Cardiol. 2008;52(19):1574-80. https:// doi.org/10.1016/j.jacc.2008.06.049

16. Ferreira VM, Piechnik SK, Dall'Armellina E, Karamitsos TD, Francis JM, Choudhury RP, et al. Non-contrast T1-mapping detects acute myocardial edema with high diagnostic accuracy: a comparison to $\mathrm{T2}$-weighted cardiovascular magnetic resonance. J Cardiovasc Magn Reson. 2012;14(1):42. https:// doi.org/10.1186/1532-429X-14-42

17. Triadyaksa P, Oudkerk M, Sijens PE. Cardiac T2* mapping : techniques and clinical applications. J Magn Reson Imaging. 2020;52(5):1340-51. https://doi.org/10.1002/jmri.27023

18. Dastidar AG, Harries I, Pontecorboli G, Bruno VD, De Garate $E$, Moret $C$, et al. Native T1 mapping to detect extent of acute and chronic myocardial infarction: comparison with late gadolinium enhancement technique. Int J Cardiovasc Imaging. 2019;35(3):517-27. https://doi.org/10.1007/s10554-018-1467-1

19. Slavin GS, Stainsby JA. True T1 mapping with SMART1Map (saturation method using adaptive recovery times for cardiac T1 mapping): a comparison with MOLLI. J Cardiovasc Magn Reson. 2013;15(Suppl 1):P3. https://doi.org/10.1186/1532429X-15-S1-P3

20. Matsumoto S, Okuda S, Yamada $Y$, Suzuki T, Tanimoto A, Nozaki A, et al. Myocardial T1 values in healthy volunteers measured with saturation method using adaptive recovery times for T1 mapping (SMART1Map) at 1.5T and 3T. Heart Vessels. 2019;34(11):1889-94. https://doi.org/10.1007/ s00380-019-01401-5

21. Ohta $Y$, Kishimoto J, Kitao S, Yunaga H, Mukai-Yatagai N, Fujii $S$, et al. Investigation of myocardial extracellular volume fraction in heart failure patients using iodine map with rapid-kV switching dual-energy CT: segmental comparison with MRI T1 mapping. J Cardiovasc Comput Tomogr. 2020;14(4):349-55. 